\title{
LLC Resonance Power Transformers Using Magnetoplated Wire
}

\author{
Yinggang $\mathrm{Bu}^{\mathrm{a},{ }^{*}}$, Masahiro Nishiyama ${ }^{\mathrm{a}}$, Tatsuya Yamamoto ${ }^{\mathrm{a}}$, Tsutomu Mizuno ${ }^{\mathrm{a}}$ \\ and Shigeaki Tsuchiya ${ }^{\mathrm{b}}$, \\ a Faculty of Engineering, Shinshu University, 4-17-1 Wakasato, Nagano, Japan \\ b TOTOKU Electric Co., Ltd., 1-11, Shinbashi 6-Chome, Minato-ku, Tokyo, Japan
}

\begin{abstract}
The use of magneto-plated wire (MPW) allows for reduction of winding loss component from the proximity effect. compared with a copper wire (COW). A MPW is a COW with a circumference that is plated with a magnetic thin film. In this paper, we clarify the basic characteristics and the efficiency characteristics of a transformer using a MPW and a COW. We found that the resistances of a primary coil made of COW and MPW at a frequency $f=500 \mathrm{kHz}$ are $1.56 \Omega$ and $1.12 \Omega$, respectively, and decreased $28 \%$. The short circuit inductances of the COW and the MPW at a frequency $f=500 \mathrm{kHz}$ are $26 \mu \mathrm{H}$ and $29.6 \mu \mathrm{H}$, respectively, and increased 13.8\%. The efficiencies of an LLC resonant converter using the COW and the MPW at a frequency $f=500 \mathrm{kHz}$ are $90.2 \%$ and $92.2 \%$, respectively, and increased $2 \%$.
\end{abstract}

Keywords: magnetoplated wire; copper wire; AC resistance; LLC resonance; transformer.

\footnotetext{
* Corresponding author. Tel.: +81 26269 5181; Fax: +81 262695215.

E-mail: buyinggang@shinshu-u.ac.jp (Y. Bu)
} 


\section{Introduction}

An LLC resonant converter is widely used in applications such as LED illumination and liquid crystal TVs, because it is highly efficient, is not loud and has few parts. Additionally, there is a demand for miniaturizing and high efficiency [1-3]. LLC resonance power transformers use leakage inductance as a resonant inductor. Therefore, these transformers tend to be large with a structure capable of separating the primary and the secondary sides. The miniaturization is indicated by an increase in frequency and an improvement in efficiency by the magnetic material from the transformer.

In improving transformer efficiency, the loss of iron and copper is problematic. The iron loss has been reduced by improving the characteristics of the core material $[4,5]$, while the copper loss has been reduced by changing the wire's shape and varying its winding form $[6,7]$. However, the reduction of the wire's AC resistance from proximity effects has not been considered.

Therefore, the authors propose the use of a magnetoplated wire (MPW) for the coil of a transformer to reduce the copper loss $[8,9]$. The MPW is a copper wire $(\mathrm{COW})$ plated with a magnetic thin film. The MPW increases inductance and decreases AC resistance because of a proximity effect in which an alternating magnetic flux flows through the magnetic thin film with greater permeability and resistivity compared with copper. Hence, the magnetic thin film 
causes the inductance to increase, and because of the proximity effect, causes the resistance to decrease, as the eddy current loss is reduced [10,11].

In this paper, the basic characteristics (resistance, inductance, mutual inductance and the coupling coefficient) of the transformer using a litz wire (LCW) with a COW and a litz wire (LMW) with a MPW are measured. Additionally, the efficiency characteristics and temperature increase of the transformer in the LLC resonant converter of the LCW and LMW are compared, and the following characteristics are described.

1) Basic characteristics of the transformer using a LCW and a LMW

2) Temperature increase and efficiency characteristics of the LLC resonant converter

2. LLC Resonant Converter Using Maganetoplated Wire

\subsection{Structures of wires}

Fig. 1 shows the structures of the COW and the MPW used for winding. The COW has a diameter of $70 \mu \mathrm{m}$ and is plated with insulating films. The MPW is a copper wire with a diameter of $70 \mu \mathrm{m}$ and is plated with magnetic thin films (Fe and $\mathrm{Ni}$ ). The $\mathrm{Fe}$ and $\mathrm{Ni}$ thin films are $0.6 \mu \mathrm{m}$ and $0.05 \mu \mathrm{m}$ thick, respectively. The Ni film is plated for ease of soldering. The thickness of the insulating film of the COW and the MPW is $8 \mu \mathrm{m}$. Each litz wire is composed of forty wires. 
2.2 Winding structure of the transformer

Fig. 2 shows the transformer's structure. Two cores of E type (JFE, material: MC2) are combined and are used for the transformer's core. The gap of the transformer is $0.1 \mathrm{~mm}$. In order not to affect the performance of the transformer, a uniform thickness film was used to control the gap in coil assembly. Fig. 3 shows a winding structure. To weaken the magnetic coupling, the primary coil and the secondary coil are separated. The thickness of the spacer is $0.25 \mathrm{~mm}$. The primary coil is wound 18 times up and down through part of the bobbin. The secondary coil is wound in a bifilar formation with three wires in parallel using the bobbin.

\subsection{Equivalent circuit of the LLC resonant converter}

Fig. 4 shows the equivalent circuit of the LLC resonant converter. The parasitic diode of the FET is connected between the drain and the source. The voltage resonance capacitor and the series resonant circuit are connected in parallel to the FET Q2. The series resonant circuit is composed of a current resonance capacitor $C_{\mathrm{r}}$, a magnetizing inductance $L_{\mathrm{m}}$ and a resonance inductor $L_{\mathrm{sh}}$. The transformer's secondary side is connected to the output through a rectifying diode D1, D2. The transformer is integrated with a resonant inductance. Hence, the resonance inductor serves as a leakage inductance for the transformer. 


\subsection{Behavior of the LLC resonant converter}

Fig. 5 shows the flow of current through the LLC resonant converter driving and Fig 6 shows the timing diagram of LLC resonant converter. Q1 and Q2 are switched alternately by the control IC. The dead time is provided to prevent the converter from conducting simultaneously. The behavior of the LLC resonant converter can be divided into four modes.

(1) Mode a-b

This mode is switched on only in Q1. An input voltage is applied to the series resonant circuit as the Q1 is switched on. Electric charge accumulates in the $C_{\mathrm{r}}$ because of the resonance behavior of $C_{\mathrm{r}}, L_{\mathrm{sh}}$ and $L_{\mathrm{m}}$. A resonant current is simultaneously released to the output through the secondary winding. Then, the voltage applied to the primary winding decreases as the electric charge $\mathrm{C}$ is released. When the voltage equals zero, the current on the secondary side is gradually reduced. Resonant current flows only on the primary side after the current of the secondary side is zero.

(2) Mode b-c

This mode corresponds to a period of dead time. The electric charge $C_{\mathrm{v}}$ is reduced gradually by a resonant current to be switched off in Q1 at the time of b. Thus, the voltage $V_{\mathrm{ds} 1}$ rise becomes gradual. Additionally, it is possible to suppress the switching loss because the current is stopped in the period in which only the circulating current is flowing. After the voltage $V_{\mathrm{ds} 2}$ reaches zero, the resonant current continues to flow in the direction of resetting the energy 
stored in $L_{\mathrm{p}}$ through the parasitic diode Q2.

(3) Mode c-d

This mode is switched on only in Q2. At the time of c, a resonant current is flowing in the direction of the negative $I_{\mathrm{ds} 2}$ in Q2. Therefore, the switching loss equals zero to achieve ZVS (Zero Voltage Switching) - ZCS (Zero Current Switching). Q2 is switched on, and the charge stored in $C_{\mathrm{r}}$ is discharged. Current flows through the series resonant circuit by the voltage charged in the $C_{\mathrm{r}}$. Simultaneously, a resonant current is released through the secondary winding. Then, the voltage applied to the primary winding decreases as the electric charge $\mathrm{C}$ is released. A resonant current flows only on the primary side after the current of the secondary side equals zero.

(4) Mode d-e

This mode corresponds to a period of dead time. $C_{\mathrm{v}}$ is gradually charged by the resonance current by switching off Q2 at the time of $\mathrm{d}$, thereby suppressing the switching loss with a more gradual increase in the voltage $V_{\mathrm{ds} 2}$. After this voltage has been raised to the input voltage, a resonance current flows in the direction in which the exciting energy is reduced. The operation returns to state a.

It is necessary to operate at a frequency higher than the resonance frequency of the series resonant circuit in this system. 
3. Impedance characteristics of the transformer

3.1 Resistance versus frequency characteristics

Fig. 7 shows the resistance versus frequency characteristics of transformer. The measurement was determined with an impedance analyzer (Agilent, 4294A). The accuracy of measurement is less than $1 \%$. Fig. 7 (a) shows the resistance characteristics of the transformer's primary coil using a LCW and a LMW. The resistances of the primary coils using the LCW and the LMW $R_{\mathrm{p}}$ at frequency $f=500 \mathrm{kHz}$ are $1.56 \Omega$ and $1.12 \Omega$, respectively; thus, the resistance of the LMW decreases $28 \%$ compared with that of the LCW. Fig. 7 (b) shows the resistance characteristics of the transformer's secondary coil using the LCW and the LMW. The resistances of the secondary coils using the LCW and the LMW $R_{\mathrm{S}}$ at frequency $f=500 \mathrm{kHz}$ are $0.026 \Omega$ and $0.019 \Omega$, respectively; thus, the resistance of the LMW decreases $27 \%$ compared with that of the LCW because the eddy current loss is reduced.

3.2 Inductance versus frequency characteristics

Fig. 8 (a) shows the primary inductance characteristics of the transformer using the LCW and the LMW. The inductances of the primary coil using the LCW and the LMW $L_{\mathrm{p}}$ at frequency $f=$ $500 \mathrm{kHz}$ are $169 \mu \mathrm{H}$ and $174 \mu \mathrm{H}$, respectively; thus, the inductance of the LMW increases $3 \%$ 
compared with that of the LCW. Fig. 8 (b) shows the secondary inductance characteristics of the transformer using the LCW and the LMW. The inductances of the secondary coil using the LCW and the LMW $L_{\mathrm{s}}$ at frequency $f=500 \mathrm{kHz}$ are $1.9 \mu \mathrm{H}$ and $2 \mu \mathrm{H}$, respectively; thus, the inductance of the LMW increases 5\% compared with that of the LCW.

3.3 Short circuit inductance and the coupling coefficient

Fig. 9 (a) shows the short circuit inductance characteristics of the transformer using the LCW and the LMW. As a condition of measurement, the secondary side of the transformer is short-circuited. The short circuit inductances of coil using the LCW and the LMW $L_{\text {sh }}$ at frequency $f=500 \mathrm{kHz}$ are $26 \mu \mathrm{H}$ and $29.6 \mu \mathrm{H}$, respectively; thus, the inductance of the LMW increases $14 \%$ compared with that of the LCW. Based on these results, it is possible to decrease the width of the spacer separating the primary coil and the secondary coil to downsize the transformer.

Fig. 9 (b) shows the coupling coefficients of the transformer using the LCW and the LMW. The coupling coefficients were calculated by substituting, into the following equation, the measured value of primary inductance $L_{\mathrm{p}}$ and short circuit inductance $L_{\mathrm{sh}}$. The coupling coefficients of the transformer using the LCW and the LMW $k$ at frequency $f=500 \mathrm{kHz}$ are 0.92 and 0.91 , respectively; thus, the coupling coefficient of the LMW decreases 0.01 compared with that of the LCW [12]. 


$$
k=\sqrt{1-\frac{L_{\mathrm{sh}}}{L_{\mathrm{p}}}}
$$

4. Efficiency and temperature of LLC resonant converter

4.1 Efficiency versus output power characteristics

Fig .10 (a) shows the input power $P_{\mathrm{i}}$ and the output power $P_{\mathrm{o}}$ versus the output current $I_{\mathrm{o}}$ characteristic of the LLC resonant converter using the LCW and LMW transformers at a drive frequency $f_{\mathrm{s}}=500 \mathrm{kHz}$ and an input voltage $V_{\mathrm{i}}=380 \mathrm{~V}$. The input power $P_{\mathrm{i}}$ and the output power $P_{\mathrm{o}}$ are measured with an electronic load (KENWOOD, PEL151-201) by the changing load $R_{\mathrm{L}}$. The input power and the output power are measured with a power meter (Yokogawa, DL9040L). No significant difference was found in the input power of the LMW and the LCW. However, the output power of the LCW was less than that of the LMW.

Fig. 10 (b) shows the efficiency $\eta$ versus the output current $I_{\mathrm{o}}$ characteristics of the LLC resonant converter using the LCW and LMW transformers. The maximum efficiencies of the LCW and the LMW are $90.2 \%$ and $92.2 \%$, respectively; thus, the efficiency of the LMW improved $2 \%$. The switching frequency was lowered to reduce the load resistance.

4.2 Temperature rise of the transformer

Fig. 11 shows the distribution of surface temperature of the transformers winding using the LCW and the LMW. The temperature inside of the winding wire is difficult to measure, therefore 
we measured the surface temperature. The temperatures of secondary winding are measured with thermography (NEC, F30). The surface temperature rises $\Delta T$ of the LCW and the LMW at the input current $I_{\mathrm{o}}=5 \mathrm{~A}$ are $52 \mathrm{deg}$. and $47 \mathrm{deg}$., respectively; thus, the temperature rise of the LMW is decreased by $5 \mathrm{deg}$. This decrease resulted from results from the greater ability of the LMW to reduce the AC resistance compared with the LCW.

\section{Conclusions}

1) Basic characteristics of transformer

The resistances of the primary coils using a LCW and a LMW $R_{\mathrm{p}}$ at frequency $f=500 \mathrm{kHz}$ are $1.56 \Omega$ and $1.12 \Omega$, respectively; thus, the resistance of the LMW decreases $28 \%$ compared with that of the LCW. Moreover, the short circuit inductances of coil using a LCW and a LMW $L_{\mathrm{sh}}$ at frequency $f=500 \mathrm{kHz}$ are $26 \mu \mathrm{H}$ and $29.6 \mu \mathrm{H}$, respectively; thus, the inductance of the LMW increases $14 \%$ compared with that of the LCW. Based on these results, it is possible to reduce the width of the spacer separating the primary coil and the secondary coil to downsize the transformer.

2) Efficiencies of the LLC resonant converter and the Temperature rise of transformer

The maximum efficiencies of the LCW and the LMW are $90.2 \%$ and $92.2 \%$, respectively; thus, the efficiency of the LMW improved $2 \%$. Moreover, the temperature rises $\Delta T$ of the LCW 
and the LMW at the input current $I_{\mathrm{o}}=5 \mathrm{~A}$ are $52 \mathrm{deg}$. and $47 \mathrm{deg}$., respectively; thus, the temperature rise of the LMW decreased 5 deg.

The above-mentioned characteristics originated from the $\mathrm{AC}$ resistance because of the proximity effect, based on which the LMW decreased and the short circuit inductance of the LMW increased.

\section{References}

[1] C. Liu, M. Wang, Y. Ji, B. Gu, J. Lai, Z. Zhao, C. Chen, C. Zheng, P. Sun and G. Cai, High-efficiency hybrid full-bridge-half-bridge converter with shared ZVS lagging leg and dual outputs in series, IEEE Trans Power Electron, 28 (2013), 849-861.

[2] J. Jung, Bifilar winding of a center-tapped transformer including integrated resonant inductance for LLC resonant converters, IEEE Trans Power Electron, 28 (2013), 615-620.

[3] H. Sarnago, O. Lucía, A. Mediano, J. M. Burdío, Analysis and design of high-efficiency resonant inverters for domestic induction heating applications, International Journal of Applied Electromagnetics and Mechanics, 44 (2014), 201-208.

[4] M. Bogs, W. Holubarsch, Design principles for high frequency power transformer materials, $J$ Phys 4 , 7 (1997), C1.117-C1.118. 
[5] A. M. Kumar, M. C. Varma, K.H. Rao, C. L. Dube, S. C. Kashyap, Development of Ni-Zn nanoferrite core material with improved saturation magnetization and DC resistivity, $J$ Magn Mater, 320 (2008),1995-2000.

[6] A. Urling, V. Niemela, G. Skutt, T. Wilson, Characterizing high-frequency effects in transformer windings - A guide to several significant articles, Proc. IEEE Applied Power Electronics Conf, (1989), 373-385.

[7] K. Yamamoto, T. M Takahashi, AC resistance of rectangular braided litz wires for switching power supplies, The Institute of Electronics, Information and Communication Engineers,(1992),253-258, (in Japanese).

[8] T. Mizuno, K. Iida, K. Matsushita, D, Yamamoto, A. Kamiya, Flyback transformer using magnetoplated wire, The paper of Technical Meeting on Magnetics, (2009) MAG-09-7, 29-33, (in Japanese).

[9] T. Mizuno, D. Yamamoto, A. Kamiya, Reduction of AC resistance of transformer using magnetoplated wire," Journal of the Japan Society of Applied Electromagnetics and Mechanics, 19 (2011), 177-182 (in Japanese).

[10] T. Mizuno, S. Enoki, T. Asahina, T. Suzuki, M. Noda, H. Shinagawa, Reduction of proximity effect in coil using magnetoplated wire, IEEE Trans. Magn., 43 (2007), 2654-2656.

[11] T. Mizuno, S. Enoki, T. Hayashi, H. Shinagawa, Extending the linearity range of eddy-current displacement sensor with magnetoplated wire, IEEE Trans. Magn., 43 (2007), 543-548. 
[12] H. Yamamura, Toroidal core encyclopedia non-english documents, CQ Press, (2006), 60-82, (in Japanese).

Caption of all figures:

Fig. 1. Litz wires using the COW and the MPW for the transformer coil (unit: $\mu \mathrm{m}$ ).

Fig. 2. Structures of the transformer (unit: $\mathrm{mm}$ ).

Fig. 3. Winding structures (unit: $\mathrm{mm}$ ).

Fig. 4. Equivalent circuit of the LLC resonant power supply.

Fig. 5. Current flow in each mode.

Fig. 6. Timing diagram of LLC resonant converter.

Fig. 7. Comparison with $R$ of the transformer vs. frequency characteristics.

Fig.8. Comparison with $L$ of transformer vs. frequency characteristics.

Fig. 9. Comparison with $L_{\mathrm{sh}}, k$ of the transformer vs. frequency characteristics.

Fig. 10. Comparison with $P_{\mathrm{i}}, P_{\mathrm{o}}$ and $\eta$ vs. output current characteristics $\left(f_{\mathrm{s}}=500 \mathrm{kHz}\right)$.

Fig. 11. Temperature rise Tr using the LCW and the LMW $\left(f_{\mathrm{s}}=500 \mathrm{kHz}, V_{\mathrm{i}}=380 \mathrm{~V}, I_{\mathrm{o}}=5 \mathrm{~A}\right.$, room temperature: $\left.25^{\circ} \mathrm{C}\right)$.

\section{Figures}




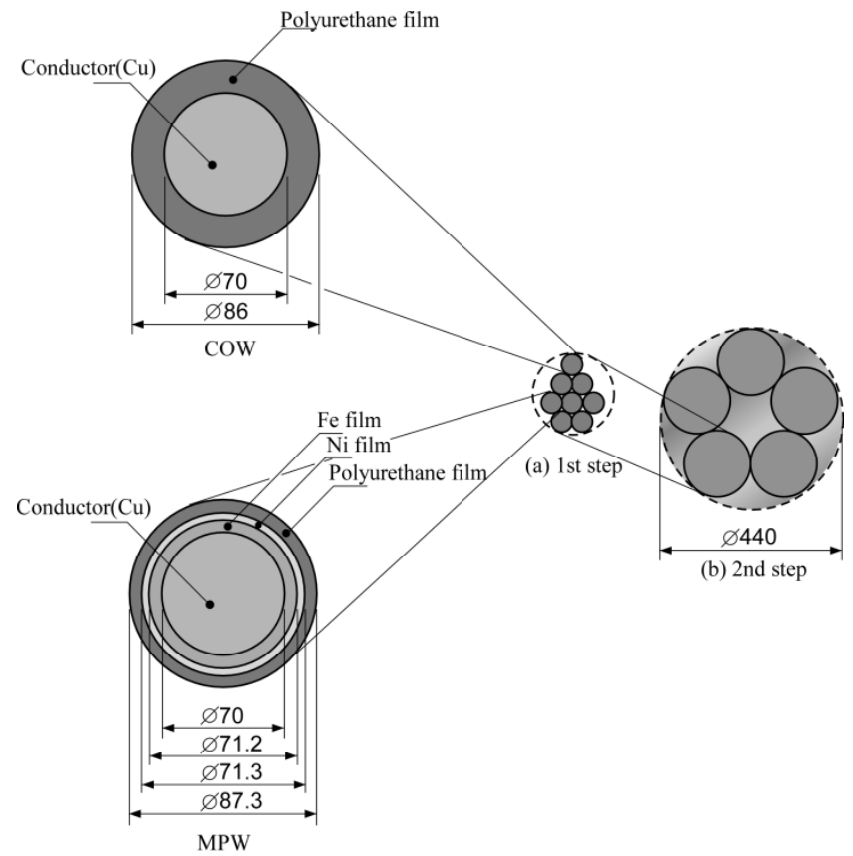

Fig. 1. Litz wires using the COW and the MPW for the transformer coil (unit: $\mu \mathrm{m}$ ). 


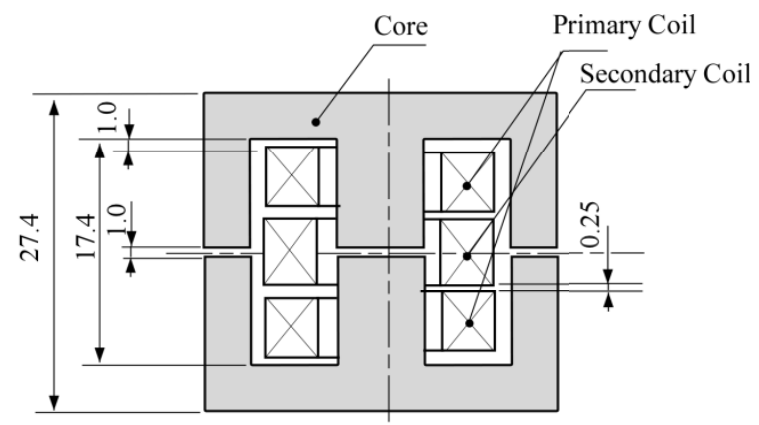

(a) Vertical cross-sectional view

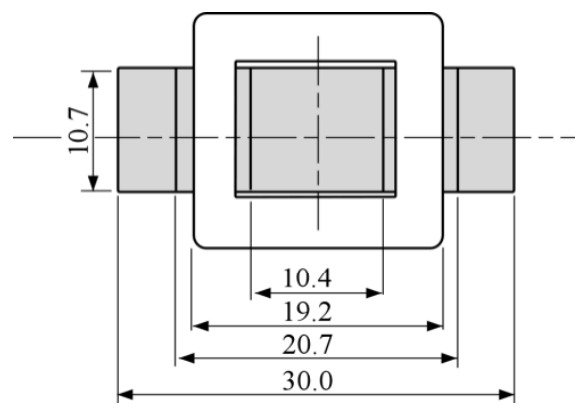

(b) Cross-section

Fig. 2. Structures of the transformer (unit: $\mathrm{mm}$ ). 


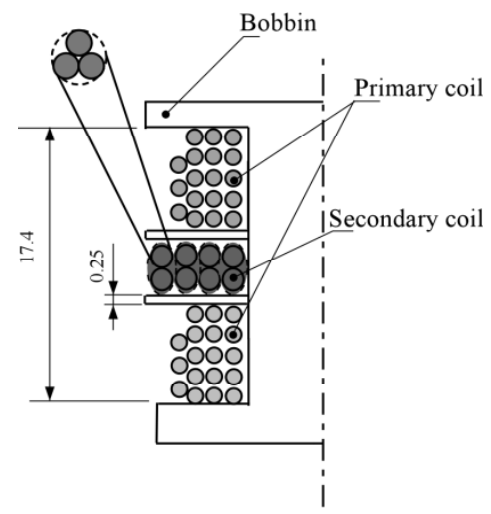

(a) Winding structure

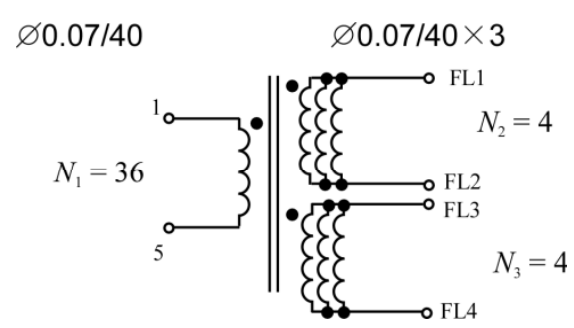

(b) Connection of winding

Fig. 3. Winding structures (unit: $\mathrm{mm}$ ). 


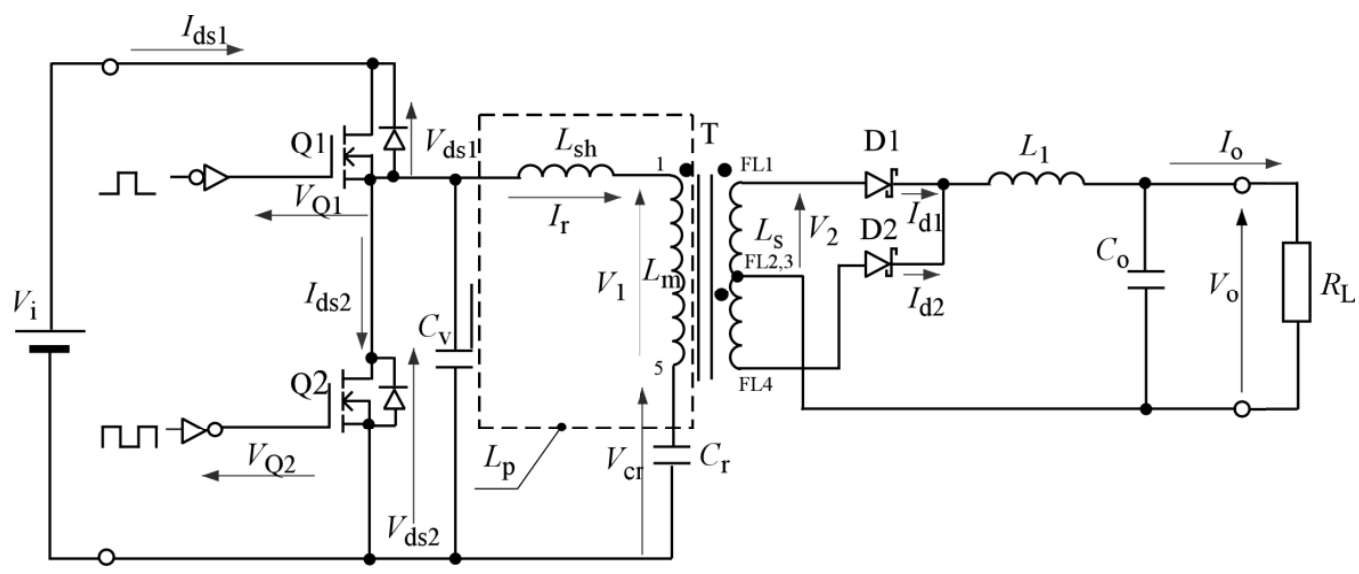

Fig. 4. Equivalent circuit of the LLC resonant power supply. 


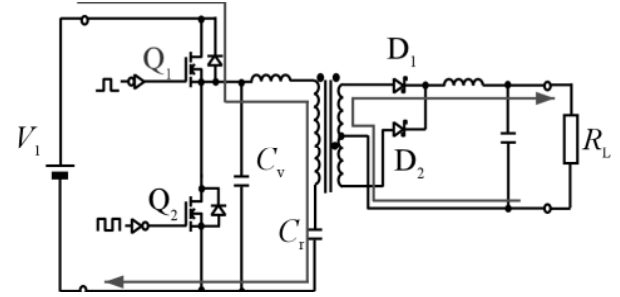

(a) Mode : a-b

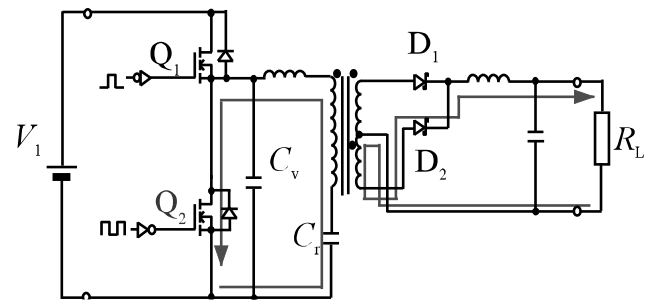

(c) Mode : c-d

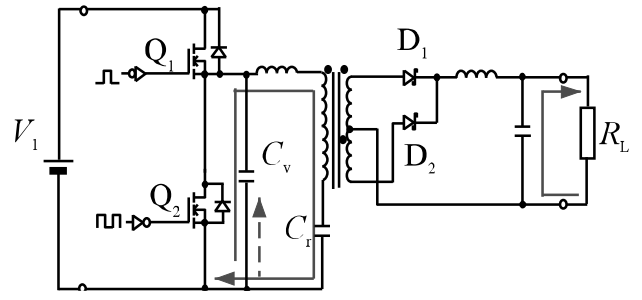

(b) Mode : b-c

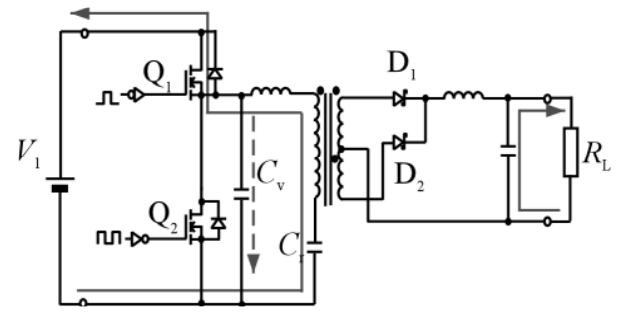

(d) Mode : d-e

Fig. 5. Current flow in each mode.

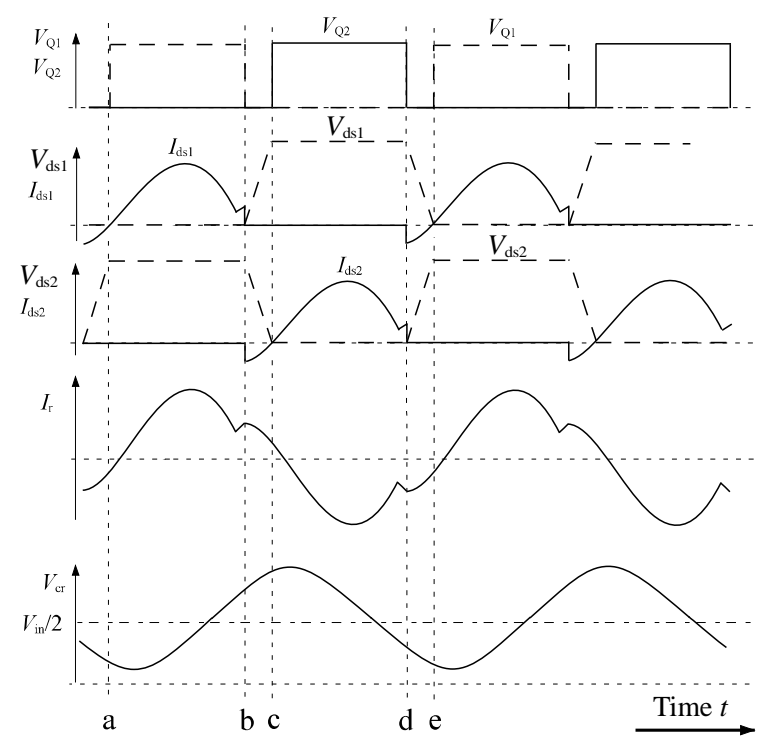

Fig. 6. Timing diagram of LLC resonant converter. 


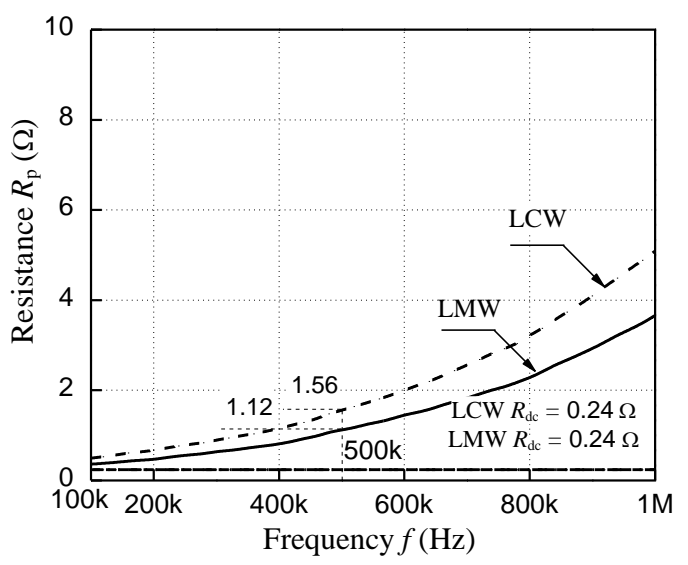

(a) Primary coil

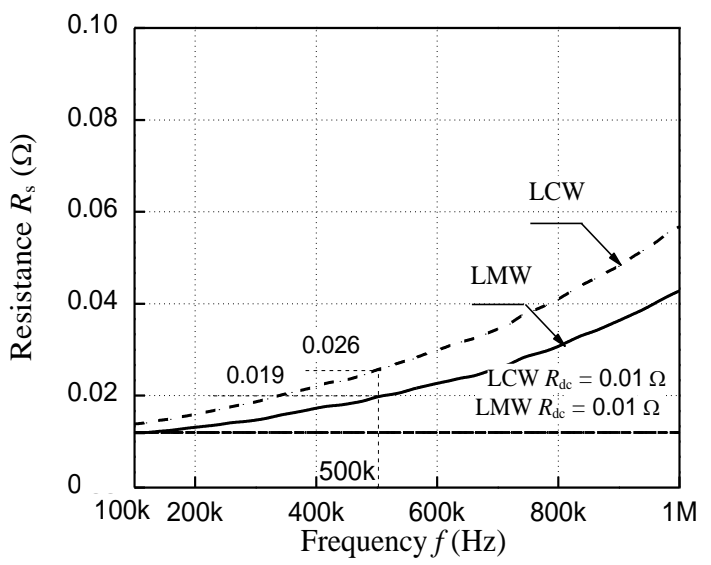

(b) Secondary coil

Fig. 7. Comparison with $R$ of the transformer vs. frequency characteristics. 


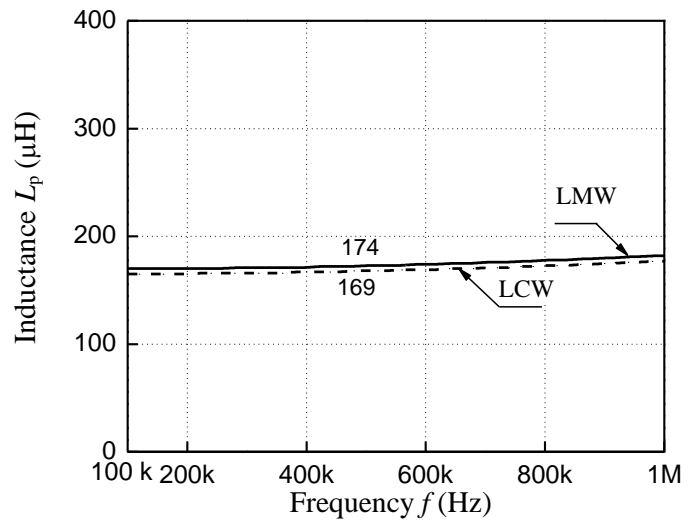

(a) Primary coil

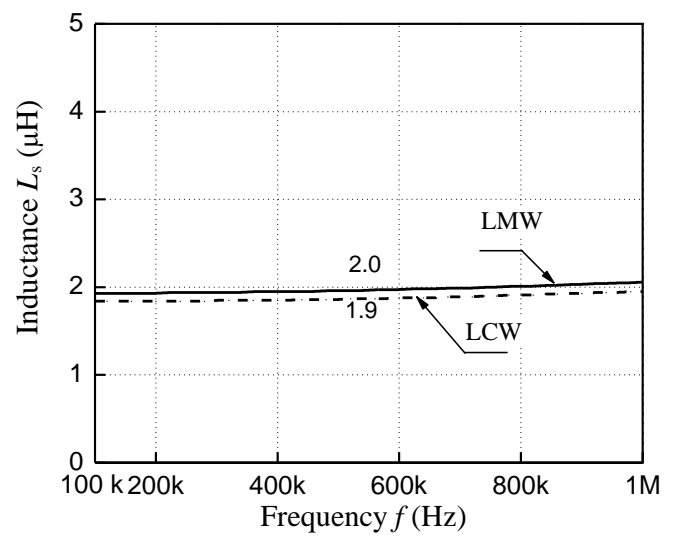

(b) Secondary coil

Fig.8. Comparison with $L$ of transformer vs. frequency characteristics. 


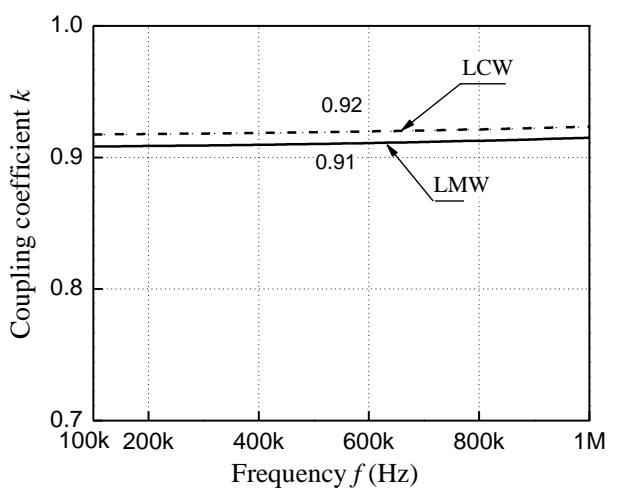

(b) Coupling coefficient

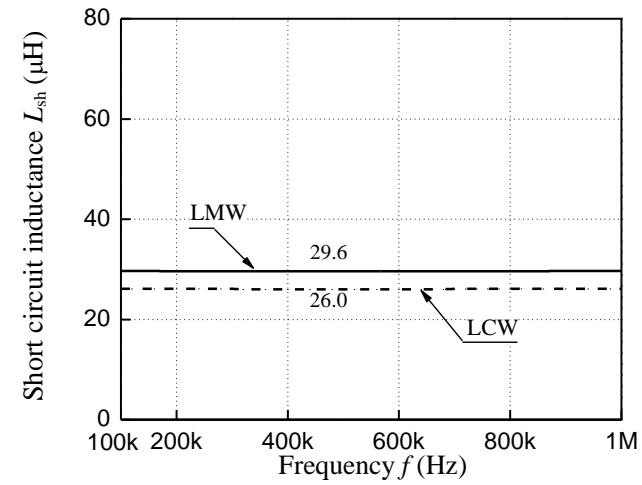

(a) Short circuit inductance

Fig. 9. Comparison with $L_{\mathrm{sh}}, k$ of the transformer vs. frequency characteristics. 


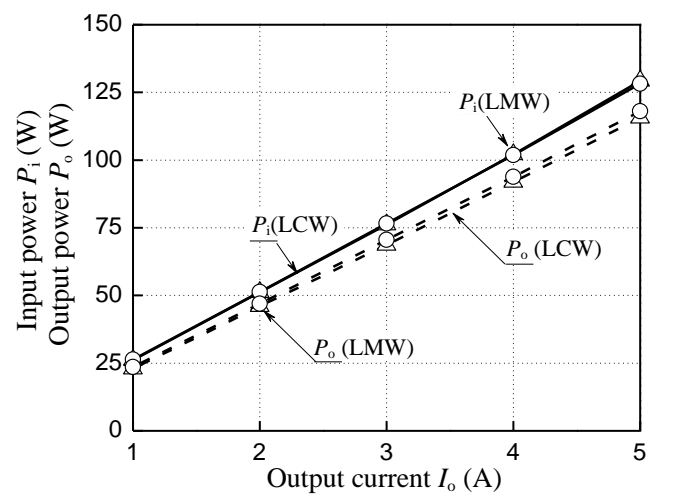

(a) $P_{\mathrm{i}}, P_{\mathrm{o}}$

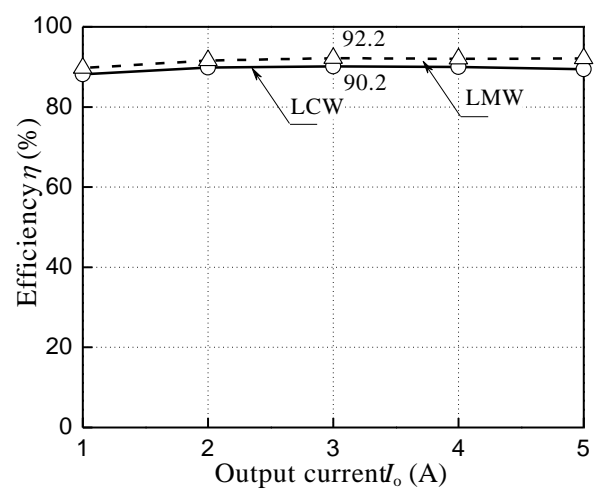

(b) $\eta$

Fig. 10. Comparison with $P_{\mathrm{i}}, P_{\mathrm{o}}$ and $\eta$ vs. output current characteristics $\left(f_{\mathrm{s}}=500 \mathrm{kHz}\right)$. 


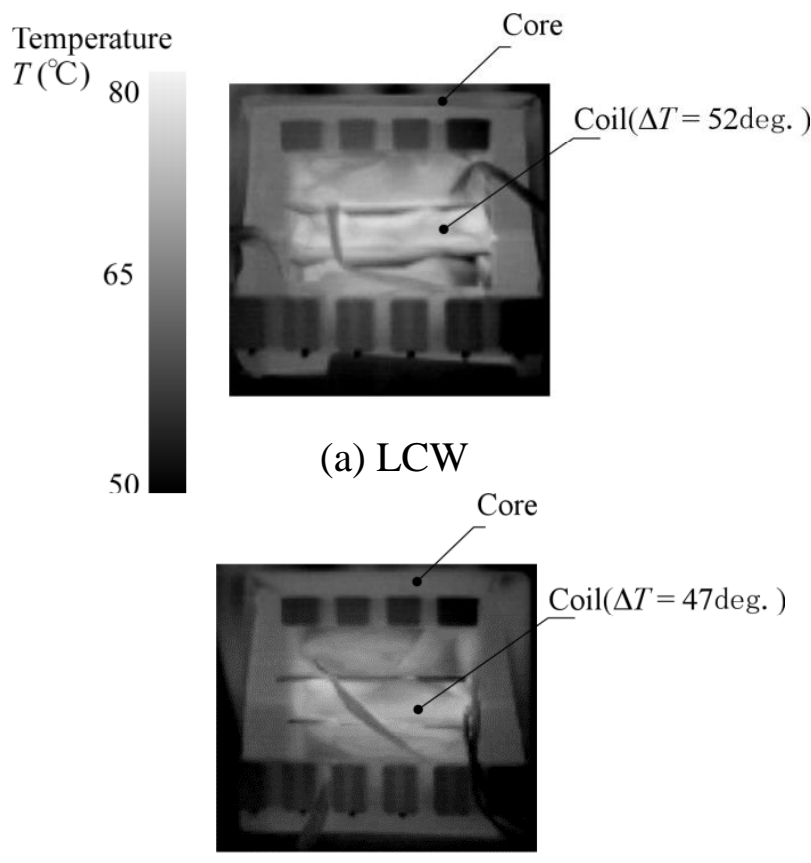

(b) LMW

Fig. 11. Temperature rise Tr using the LCW and the LMW $\left(f_{\mathrm{s}}=500 \mathrm{kHz}, V_{\mathrm{i}}=380 \mathrm{~V}, I_{\mathrm{o}}=5 \mathrm{~A}\right.$, room temperature: $\left.25^{\circ} \mathrm{C}\right)$. 